\title{
Glucagon-Like Peptide-1 Receptor Agonists and Cardiovascular Events: A Meta-Analysis of Randomized Clinical Trials
}

\author{
Matteo Monami, ${ }^{1}$ Francesco Cremasco, ${ }^{2}$ Caterina Lamanna, ${ }^{2}$ Claudia Colombi, ${ }^{2}$ \\ Carla Maria Desideri, ${ }^{1}$ Iacopo Iacomelli, ${ }^{1}$ Niccolò Marchionni, ${ }^{1}$ and Edoardo Mannucci ${ }^{1,2,3}$ \\ ${ }^{1}$ Section of Geriatric Cardiology and Medicine, Department of Cardiovascular Medicine, University of Florence and Careggi Teaching \\ Hospital, 50141 Florence, Italy \\ ${ }^{2}$ Diabetes Agency, University of Florence and Careggi Teaching Hospital, 50141 Florence, Italy \\ ${ }^{3}$ Section of Geriatric Cardiology and Medicine, Department of Cardiovascular Medicine, Azienda Ospedaliero-Universitaria Careggi, \\ Pieraccini Avenue 18, 50141 Florence, Italy
}

Correspondence should be addressed to Matteo Monami, mmonami@libero.it

Received 26 December 2010; Accepted 22 February 2011

Academic Editor: Giovanni Di Pasquale

Copyright (C) 2011 Matteo Monami et al. This is an open access article distributed under the Creative Commons Attribution License, which permits unrestricted use, distribution, and reproduction in any medium, provided the original work is properly cited.

Objective. Data from randomized clinical trials with metabolic outcomes can be used to address concerns about potential issues of cardiovascular safety for newer drugs for type 2 diabetes. This meta-analysis was designed to assess cardiovascular safety of GLP-1 receptor agonists. Design and Methods. MEDLINE, Embase, and Cochrane databases were searched for randomized trials of GLP-1 receptor agonists (versus placebo or other comparators) with a duration $\geq 12$ weeks, performed in type 2 diabetic patients. Mantel-Haenszel odds ratio with 95\% confidence interval (MH-OR) was calculated for major cardiovascular events (MACE), on an intention-to-treat basis, excluding trials with zero events. Results. Out of 36 trials, 20 reported at least one MACE. The MHOR for all GLP-1 receptor agonists was $0.74(0.50-1.08), P=.12(0.85(0.50-1.45), P=.55$, and $0.69(0.40-1.22), P=.20$, for exenatide and liraglutide, resp.). Corresponding figures for placebo-controlled and active comparator studies were 0.46 ( $0.25-$ $0.83), P=.009$, and $1.05(0.63-1.76), P=.84$, respectively. Conclusions. To date, results of randomized trials do not suggest any detrimental effect of GLP-1 receptor agonists on cardiovascular events. Specifically designed longer-term trials are needed to verify the possibility of a beneficial effect.

\section{Introduction}

Cardiovascular safety is a growing concern for drugs used for chronic conditions, such as diabetes. Among glucoselowering agents, sulfonylureas $[1,2]$, insulin $[3,4]$, and thiazolidinediones [5-7], have been suspected of adverse cardiovascular effects, although some of those preoccupations have not been confirmed [8-11]. Following these concerns, the Food and Drug Administration issued a guidance for companies submitting new chemical entities as treatments for type 2 diabetes, requiring that, either in phase II-III trials, or in a subsequent phase IV specifically designed randomized clinical trial, a sufficient amount of information is collected so as to exclude a risk increase of over $30 \%$ (i.e., the upper limit—two-sided—of $95 \%$ confidence interval for major cardiovascular events, in comparison with placebo and/or other treatments, should not exceed 1.30; http://www.fda .gov/downloads/Drugs/GuidanceComplianceRegulatoryInformation/Guidances/UCM071627.pdf).

Two GLP-1 receptor agonists (exenatide and liraglutide) have been approved for human use, and several others are currently under clinical development. It has been observed that chronic stimulation of GLP-1 receptors could produce beneficial effects on several cardiovascular risk factors [12]; furthermore, preliminary data on humans suggest that GLP1 could have direct effects on myocardial function [13]. However, no major trial assessing the effects of GLP-1 receptor agonists on cardiovascular morbidity and mortality is available to date, nor will it be for a few years. In the meantime, the information on incident cases recorded as adverse 


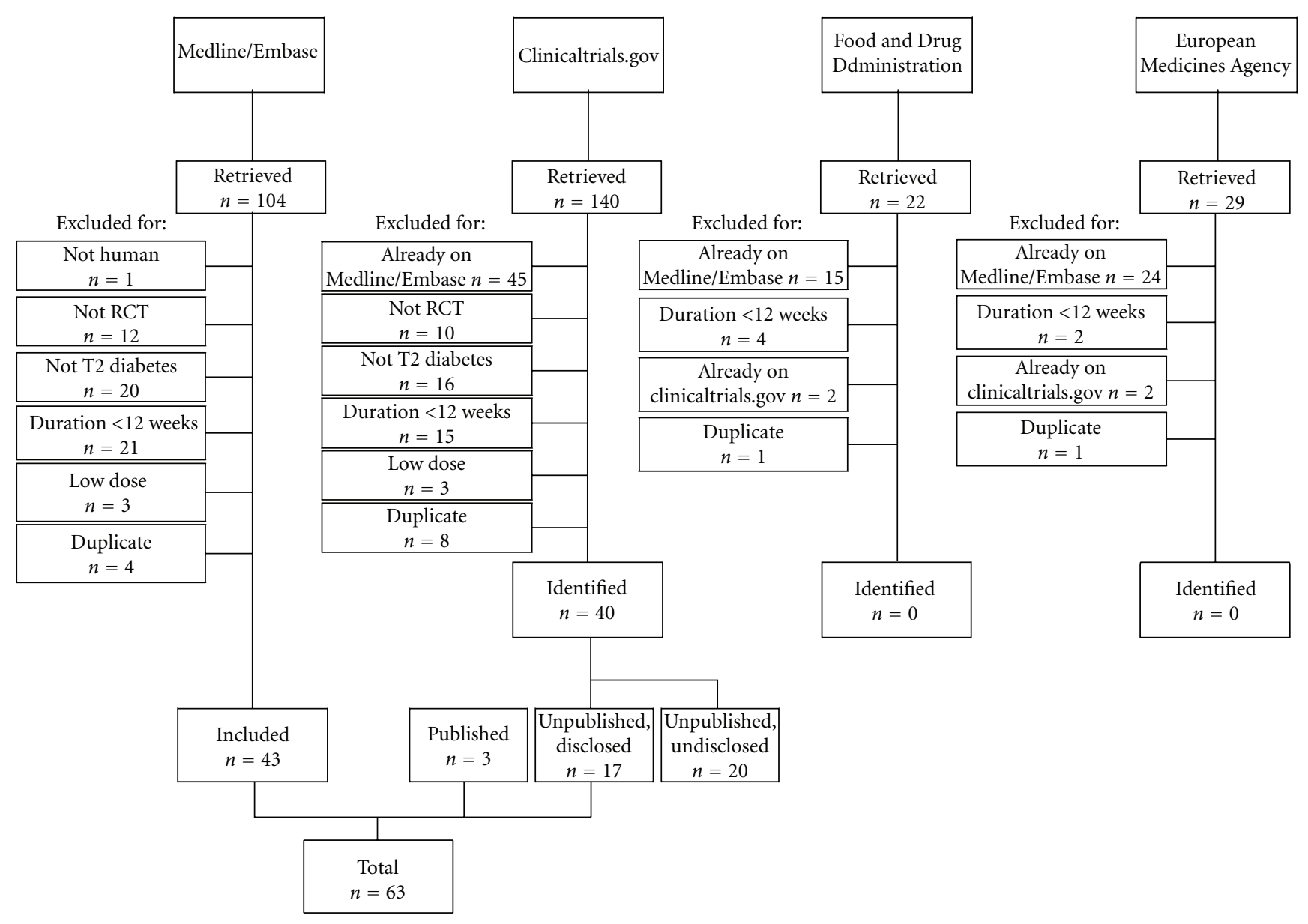

Figure 1: Trial flow diagram. RCT: randomized clinical trial; T2: type 2.

events during trials designed for metabolic endpoints could provide some hints on the possible cardiovascular profile of these drugs. This meta-analysis was designed to assess the effect of GLP-1 receptor agonists, compared with placebo or active hypoglycemic drugs, on major cardiovascular events in type 2 diabetic patients, as derived from randomized controlled trials.

\section{Research Design and Methods}

2.1. Data Sources and Searches. An extensive Medline, Embase, and Cochrane database search for "exenatide," "liraglutide," "albiglutide," "taspoglutide," "lixisenatide," and "semaglutide" was performed, collecting all randomized clinical trials on humans up to November 1th, 2010. The identification of relevant abstracts, the selection of studies based on the criteria described above, and the subsequent data extraction were performed independently by two of the authors (E. Mannucci and M. Monami), and conflicts resolved by the third investigator (N. Marchionni). Completed but still unpublished trials were identified through a search of http:// www.clinicaltrials.gov/ website. Food and Drug Administration (FDA, http://www.fda.gov/) and European Medicines Agency (EMEA, http://www.ema.europa.eu/) reviews of ap- proved drugs, as well as published information provided to FDA in response to queries during the approval process, were also searched for retrieval of unpublished trials.

2.2. Study Selection. A meta-analysis was performed including all randomized clinical trials with a duration of at least 12 weeks, either with a cross-over or a parallel series design, enrolling patients with type 2 diabetes, comparing glucagonlike peptide-1 (GLP-1) receptor agonists with placebo or active drugs (oral hypoglycemic agents and/or insulin) of other classes. Trials enrolling nondiabetic, or type 1 diabetic, subjects were also excluded. No review protocol was published elsewhere.

2.3. Data Extraction and Quality Assessment. Results of unpublished trials (characteristics of patients enrolled, treatments, and major cardiovascular events) were retrieved, if available, on http://www.clinicaltrials.gov/, http://www.novonordisk-trials.com/website/content/trial-results.aspx, http: //www.lillytrials.com/results/results.html, or http://www.clinicalstudyresults.org/; Food and Drug Administration (FDA, http://www.fda.gov/) and European Medicines Agency (EMEA, http://www.ema.europa.eu/) reviews of approved drugs, as well as published information provided to FDA in 
TABLE 1: Characteristics of the unpublished and undisclosed studies.

\begin{tabular}{lcccccc}
\hline Study & $\begin{array}{c}\text { Number of } \\
\text { patients } \\
\text { planned }\end{array}$ & Comparator & Add-on to & $\begin{array}{c}\text { Trial duration } \\
\text { (wks) }\end{array}$ & Design & Study end date* \\
\hline $\begin{array}{l}\text { Exenatide } \\
\text { NCT00434954 }\end{array}$ & 488 & Aspart & Metformin & 26 & PS, DB & August 2009 \\
$\begin{array}{l}\text { Liraglutide } \\
\text { NCT00696657 }\end{array}$ & 415 & Placebo & None & 12 & PS, DB & February 2009 \\
$\begin{array}{l}\text { Taspoglutide } \\
\text { NCT00809705 }\end{array}$ & 60 & Placebo & None & 12 & PS, DB & February 2010 Hoff-Roche \\
\hline
\end{tabular}

PS: parallel series; DB: double blind; Hoff. Roche: Hoffman-La Roche; Novo: Novo Nordisk.

Study name

Nauck et al. [31]

Heine et al. [34]

Russell-Jones\# et al. [44]

NCT00360334 et al. [33]

Diamant et al. [36]

NCT00393718 et al. [33]

Pratley et al. [50]

Nauck\# et al. [47]

NCT00614120 et al. [33]

Garber et al. [49]

Davis et al. [37]

Versus active comparators (overall)

Kendall et al. [28]

Russell-Jones et al. [44]

Rosenstock et al. [17]

Marre et al. [46]

Buse et al. [27]

DeFronzo et al. [26]

Kaku et al. [43]

Gao et al. [21]

Nauck et al. [47]

Zinman et al. [45]

Bergenstal et al. [39]

Marre\# et al. [46]

Bergenstal et al. [39]\#

Versus placebo (overall)

Overall
Statistics for each study

$\begin{array}{ccccc}\begin{array}{c}\text { MH odds } \\ \text { ratio }\end{array} & \begin{array}{c}\text { Lower } \\ \text { limit }\end{array} & \begin{array}{c}\text { Upper } \\ \text { limit }\end{array} & Z \text { value } & P \text { value } \\ 1.833 & 0.667 & 5.036 & 1.176 & .24 \\ 1.588 & 0.376 & 6.713 & 0.629 & .529 \\ 2.555 & 0.4911 & 3.304 & 1.114 & .265 \\ 0.983 & 0.136 & 7.096 & -0.017 & .986 \\ 3 & 0.122 & 74.023 & 0.672 & .502 \\ 0.652 & 0.144 & 2.954 & -0.556 & .579 \\ 0.244 & 0.022 & 2.704 & -1.15 & .25 \\ 0.497 & 0.099 & 2.492 & -0.85 & .396 \\ 0.494 & 0.082 & 2.976 & -0.769 & .442 \\ 0.496 & 0.069 & 3.542 & -0.699 & .484 \\ 1.523 & 0.059 & 39.477 & 0.253 & .8 \\ 1.054 & 0.633 & 1.756 & 0.203 & .839 \\ 0.501 & 0.174 & 1.445 & -1.279 & .201 \\ 0.822 & 0.193 & 3.503 & -0.265 & .791 \\ 0.053 & 0.003 & 1.042 & -1.933 & .053 \\ 0.243 & 0.04 & 1.469 & -1.541 & .123 \\ 0.245 & 0.022 & 2.728 & -1.144 & .253 \\ 0.25 & 0.022 & 2.787 & -1.127 & .26 \\ 0.497 & 0.031 & 8.043 & -0.492 & .623 \\ 0.329 & 0.013 & 8.119 & -0.68 & .497 \\ 2.017 & 0.113 & 36.059 & 0.477 & .633 \\ 1.485 & 0.06 & 36.644 & 0.242 & .809 \\ 0.145 & 0.007 & 2.823 & -1.275 & .202 \\ 2.35 & 0.121 & 45.668 & 0.564 & .572 \\ 0.344 & 0.014 & 8.500 & -0.652 & .514 \\ 0.459 & 0.255 & 0.826 & -2.599 & .009 \\ 0.737 & 0.501 & 1.083 & -1.552 & .121\end{array}$

$\mathrm{MH}$ odds ratio and 95\% CI

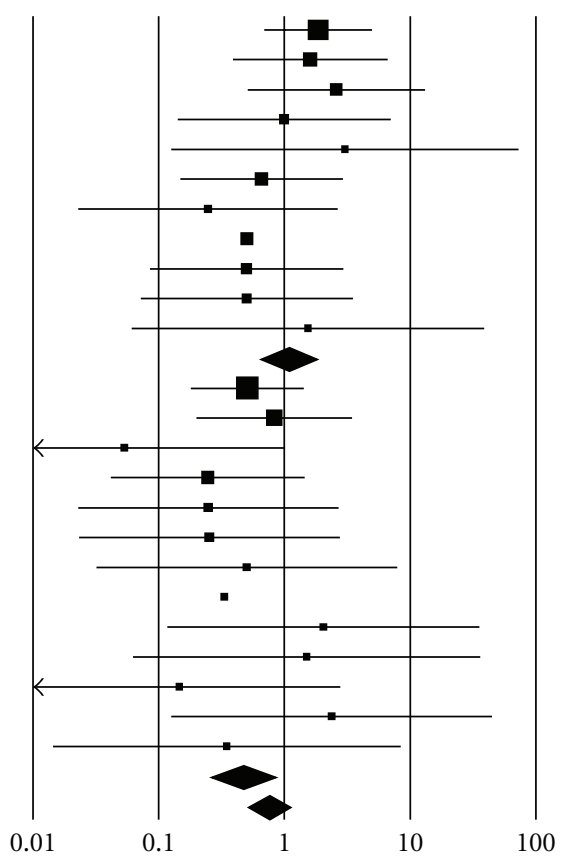

Fav ours GLP-1RA Fav ours comparator

Figure 2: Effect of GLP-1 receptor agonists on fatal and nonfatal major cardiovascular events (MACE). Forest plot of individual studies. GLP-1 RA: glucagon-like peptide-1 receptor agonists. "Studies with multiple comparators.

response to queries during the approval process, were also searched for retrieval of unpublished information. All those sources were also used to complete information on results of published trials, when not reported in publications. For all published trials, results reported in papers were used as the primary source of information, when available.

The quality of trials was assessed using some of the parameters proposed by Jadad et al. [14]. The score was not used as a criterion for the selection of trials whereas some items were used only for descriptive purposes.

2.4. Data Synthesis and Analysis. The principal outcome was the effect of GLP-1 receptor agonists, compared with other hypoglycemic agents or placebo, on major cardiovascular events (MACE) as defined in the list provided by FDA for this purpose (http://www.fda.gov/downloads/AdvisoryCommittees/CommitteesMeetingMaterials/Drugs/EndocrinologicandMetabolicDrugsAdvisoryCommittee/UCM148659.pdf), including cardiovascular death, nonfatal myocardial infarction and stroke, and hospitalizations due to acute coronary syndromes and/or heart failure.

Predefined separate analyses were performed for trials with different GLP-1 receptor agonists, whenever possible.

Mantel-Haenszel odds ratio with $95 \%$ confidence interval (MH-OR) was calculated for each of the events defined above, on an intention-to-treat basis, excluding trials with 
TABLE 2: Characteristics of the studies included in the meta-analysis.

\begin{tabular}{|c|c|c|c|c|c|c|c|}
\hline Study* (ref.) & $\begin{array}{l}\text { NCT/FDA- } \\
\text { reference }\end{array}$ & Add-on to & $\begin{array}{l}\text { Description of } \\
\text { randomization }\end{array}$ & $\begin{array}{l}\text { Description of } \\
\text { allocation }\end{array}$ & $\begin{array}{c}\text { Description of } \\
\text { blinding }\end{array}$ & $\begin{array}{l}\text { Reporting of } \\
\text { drop-out }\end{array}$ & $\begin{array}{c}\text { Intention- } \\
\text { to-treat }\end{array}$ \\
\hline \multicolumn{8}{|c|}{ Albiglutide versus placebo } \\
\hline Rosenstock et al. [19] & NCT00518115 & None/Metf. & $\mathrm{NA}$ & NA & A & A & Yes \\
\hline \multicolumn{8}{|c|}{ Exenatide versus placebo } \\
\hline Gill et al. [20] & NCT00516074 & Metf./TZD & NA & NA & A & A & Yes \\
\hline Kadowaki et al. [21] & NCT00382239 & Sulfonylurea & $\mathrm{A}$ & NA & A & A & Yes \\
\hline Zinman et al. [22] & NCT00099320 & TZD & A & A & A & A & Yes \\
\hline Gao et al. [23] & NCT00324363 & SU + Metf. & A & NA & A & A & Yes \\
\hline DeFronzo et al. [24] & NCT00135330 & Rosiglitazone & A & NA & OL & A & Yes \\
\hline Apovian et al. [25] & $\mathrm{NR}$ & Multiple & A & A & A & A & Yes \\
\hline Moretto et al. [26] & NCT00381342 & None & A & A & A & A & Yes \\
\hline Liutkus et al. [27] & NR & $\begin{array}{c}\text { Metf/TZD + } \\
\text { Met }\end{array}$ & A & $\mathrm{A}$ & A & A & Yes \\
\hline DeFronzo et al. [28] & NCT00039013 & Metformin & A & NA & A & A & Yes \\
\hline Buse et al. [29] & NCT00039026 & Sulfonylurea & A & NA & A & A & Yes \\
\hline Kendall et al. [30] & NCT00035984 & SU + Metf. & NA & NA & A & A & Yes \\
\hline \multicolumn{8}{|c|}{ Exenatide versus rosiglitazone } \\
\hline DeFronzo ${ }^{\#}$ et al. [24] & NCT00135330 & None & A & NA & $\mathrm{OL}$ & A & Yes \\
\hline \multicolumn{8}{|c|}{ Exenatide versus glibenclamide } \\
\hline Derosa et al. [31] & NCT00135330 & None & A & NA & $\mathrm{OL}$ & A & Yes \\
\hline \multicolumn{8}{|c|}{ Exenatide versus BiAsp 30/70 } \\
\hline Bergenstal et al. [32] & NCT00097877 & SU + Metf. & A & A & $\mathrm{OL}$ & A & Yes \\
\hline Nauck et al. [33] & NCT00082407 & SU + Metf. & A & A & OL & A & Yes \\
\hline \multicolumn{8}{|c|}{ Exenatide versus glargine } \\
\hline Barnett et al. [34] & NCT00099619 & SU + Metf. & A & A & OL & A & Yes \\
\hline NCT00360334 [35] & NCT00360334 & OAD & NR & NR & $\mathrm{OL}$ & A & Yes \\
\hline Heine et al. [36] & NCT00082381 & SU + Metf. & A & A & OL & A & Yes \\
\hline Bunck et al. [37] & NCT00097500 & Metformin & A & NA & $\mathrm{OL}$ & A & Yes \\
\hline Diamant et al. [38] & NCT00641056 & $\begin{array}{c}\text { SU + } \\
\text { Metf./Metf }\end{array}$ & A & A & $\mathrm{OL}$ & A & Yes \\
\hline \multicolumn{8}{|c|}{ Exenatide versus insulin } \\
\hline Davis et al. [39] & NCT00099333 & SU/Metf. & NA & NA & $\mathrm{OL}$ & A & Yes \\
\hline \multicolumn{8}{|c|}{ Exenatide LAR versus placebo } \\
\hline Kim et al. [40] & NCT00103935 & Metf./None & A & A & A & A & Yes \\
\hline \multicolumn{8}{|c|}{ Exenatide LAR versus pioglitazone } \\
\hline Bergenstal et al. [41] & NCT00637273 & None & A & A & A & A & Yes \\
\hline \multicolumn{8}{|c|}{ Exenatide LAR versus sitagliptin } \\
\hline Bergenstal et al. [41] ${ }^{\#}$ & NCT00637273 & None & A & A & A & A & Yes \\
\hline \multicolumn{8}{|c|}{ Liraglutide versus placebo } \\
\hline Madsbad et al. [42] & FDA_1310 & None & NA & NA & A & A & Yes \\
\hline Vilsbøll [43] & NCT00154401 & None & NA & NA & A & A & Yes \\
\hline Seino et al. [44] & FDA_1334 & None & A & A & A & A & Yes \\
\hline Kaku et al. [45] & NCT00395746 & Sulfonylurea & NA & NA & NA & NA & Yes \\
\hline Russell-Jones et al. [46] & NCT00331851 & SU + Metf. & A & A & A & A & Yes \\
\hline Zinman et al. [47] & NCT00333151 & Metf. + TZD & A & $\mathrm{A}$ & A & A & Yes \\
\hline Marre et al. [48] & NCT00318422 & Sulfonylurea & NA & NA & A & A & Yes \\
\hline Nauck et al. [49] & NCT00318461 & Metformin & A & A & A & A & Yes \\
\hline
\end{tabular}


Table 2: Continued.

\begin{tabular}{|c|c|c|c|c|c|c|c|}
\hline Study* (ref.) & $\begin{array}{l}\text { NCT/FDA- } \\
\text { reference }\end{array}$ & Add-on to & $\begin{array}{l}\text { Description of } \\
\text { randomization }\end{array}$ & $\begin{array}{c}\text { Description of } \\
\text { allocation }\end{array}$ & $\begin{array}{l}\text { Description of } \\
\text { blinding }\end{array}$ & $\begin{array}{c}\text { Reporting of } \\
\text { drop-out }\end{array}$ & $\begin{array}{c}\text { Intention- } \\
\text { to-treat }\end{array}$ \\
\hline \multicolumn{8}{|c|}{ Liraglutide versus metformin } \\
\hline Feinglos et al. [50] & NR & None & NA & NA & NA & A & No \\
\hline \multicolumn{8}{|c|}{ Liraglutide versus rosiglitazone } \\
\hline Marre $^{\#}$ et al. [48] & NCT00318422 & Sulfonylurea & NA & NA & A & A & Yes \\
\hline \multicolumn{8}{|c|}{ Liraglutide versus glimepiride } \\
\hline Madsbad $^{\#}$ et al. [42] & NR & None & NA & NA & OL & A & Yes \\
\hline NCT00614120 [35] & NCT00614120 & Metformin & NR & NR & $\mathrm{OL}$ & NR & NR \\
\hline Nauck $^{\#}$ et al. [49] & NCT00318461 & Metformin & A & A & OL & A & Yes \\
\hline Garber et al. [51] & NCT00294723 & None & A & A & OL & A & Yes \\
\hline \multicolumn{8}{|c|}{ Liraglutide versus glibenclamide } \\
\hline NCT00393718 [35] & NCT00393718 & None & NR & NR & OL & NR & NR \\
\hline \multicolumn{8}{|c|}{ Liraglutide versus sitagliptin } \\
\hline Pratley et al. [52] & NCT00700817 & None & A & A & OL & A & Yes \\
\hline \multicolumn{8}{|c|}{ Liraglutide versus glargine } \\
\hline Russell-Jones ${ }^{\#}$ et al. [46] & NCT00331851 & SU + Metf. & A & A & A & A & Yes \\
\hline
\end{tabular}

zero events. A random effect model was used because of the impossibility of a reliable assessment of heterogeneity, due to the small number of events in each trial [15]. Publication bias was not assessed, considering that the small number of adverse cardiovascular events in each study was irrelevant for the decision to publish trials with metabolic endpoints. The main expected bias is represented by the fact that the trials included were designed for noncardiovascular (metabolic) endpoint; this means that cardiovascular events were reported only as adverse events, without any systematic screening or predefined diagnostic criteria. The metaanalysis was reported following the PRISMA checklist [16]. All analyses were performed using Comprehensive Metaanalysis Version 2, Biostat (Englewood, NJ, USA) and SPSS 16.0 .

This research was performed independently of any funding, as part of the institutional activity of the investigators.

\section{Results}

The trial flow is summarized in Figure 1. A total of 36 trials, 3 of which unpublished, were retrieved. Information on major cardiovascular events was reported in 33 trials, 20 of which with at least one event. The analysis on MACE was therefore performed on 20 trials, enrolling 6,490 and 3,995 patients (3.467 and 2.172 patient* years) in the GLP-1 receptor agonist and comparator groups, respectively. The characteristics of the retrieved trials, and of those which resulted to be complete but were undisclosed, or did not report information on MACE, are summarized in Tables 1, 2, and 3.
The total number of patients with events was $65(0.01 \%)$ and $49(0.01 \%)$ in the GLP-1 receptor agonists and comparator groups, respectively. Treatment with the experimental drugs was not associated with an increased incidence of MACE (MH-OR.0.74 (0.50-1.08); $P=.12)$. A significant reduction of cardiovascular events with GLP-1 receptor agonists was observed in placebo-controlled trials but not in studies versus active comparators (Figure 2). No consistent pattern suggesting differences between exenatide and liraglutide emerged across analyses. In comparisons with insulin (5 trials with events) and sulfonylureas (4 trials with events), the $\mathrm{MH}-\mathrm{OR}$ for GLP-1 receptor agonists was 1.77 (0.91-3.44), $P=.09$, and $0.49(0.22-1.10), P=.085$, respectively.

All-cause mortality was reported in 33 trials, 9 of which with at least one event ( 8 and 7 ) in GLP-1 receptor agonists and comparator, respectively; $\mathrm{MH}-\mathrm{OR}$ for experimental drugs was 0.67 [0.26-1.78], $P=.43$.

\section{Conclusions}

The reduction of cardiovascular morbidity and mortality is one of the main aims of long-term treatment of hyperglycemia in type 2 diabetes. Therefore, the possibility of an increased cardiovascular risk associated with some hypoglycemic treatments [1,3-7] is almost paradoxical. Although some data on adverse cardiovascular effects of specific drugs were not confirmed by subsequent investigations [8-11], the concerns of health authorities about the safety of new compounds appear to be justified (http://www.fda.gov/downloads/Drugs/GuidanceComplianceRegulatoryInformation/Guidances/UCM071627.pdf). 
TABLE 3: Moderators and outcome variables in individual studies included in the meta-analysis.

\begin{tabular}{|c|c|c|c|c|c|c|c|c|c|}
\hline Study (ref.) & $\begin{array}{c}\text { Number of } \\
\text { patients } \\
\text { (ID/C) }\end{array}$ & $\begin{array}{c}\text { Trial } \\
\text { duration } \\
(\text { wks })\end{array}$ & Age (ys) & $\begin{array}{c}\text { Duration of } \\
\text { DM (ys) }\end{array}$ & $\begin{array}{c}\text { HbAlc/FPG } \\
\text { baseline } \\
(\% / \mathrm{mmol} / \mathrm{L})\end{array}$ & $\begin{array}{c}\text { BMI } \\
\text { baseline } \\
\left(\mathrm{Kg} / \mathrm{m}^{2}\right)\end{array}$ & $\begin{array}{c}\text { MACE } \\
(\mathrm{n}, \mathrm{ID} / \mathrm{C})\end{array}$ & $\begin{array}{l}\text { All-cause } \\
\text { mortality } \\
(\mathrm{n}, \mathrm{ID} / \mathrm{C})\end{array}$ & $\begin{array}{c}\text { Cardiovasc. } \\
\text { mortality } \\
(\mathrm{n}, \mathrm{ID} / \mathrm{C}) \\
\end{array}$ \\
\hline \multicolumn{10}{|c|}{ Albiglutide versus placebo } \\
\hline Rosenstock et al. [19] & $128 / 50$ & 16 & 54 & 5 & $8.0 / 9.7$ & 32.0 & $0 / 3$ & NR/NR & NR/NR \\
\hline \multicolumn{10}{|l|}{ Exenatide versus placebo } \\
\hline Gill et al. [20] & $27 / 25$ & 12 & 55 & NR & 7.3/NR & NR & $0 / 0$ & $0 / 0$ & $0 / 0$ \\
\hline Kadowaki et al. [21] & $115 / 40$ & 12 & 59 & 11 & $8.0 / 9.1$ & 25.9 & $0 / 0$ & $0 / 0$ & $0 / 0$ \\
\hline Zinman et al. [22] & $121 / 112$ & 16 & 56 & 8 & $7.9 / 8.9$ & 34.0 & $0 / 0$ & $0 / 0$ & $0 / 0$ \\
\hline Gao et al. [23] & $234 / 232$ & 16 & 55 & 8 & $8.3 / 9.3$ & 26.2 & $0 / 1$ & $0 / 0$ & $0 / 0$ \\
\hline DeFronzo et al. [24] & $47 / 45$ & 20 & 56 & NR & 7.9/NR & NR & $0 / 0$ & $0 / 0$ & $0 / 0$ \\
\hline Apovian et al. [25] & $96 / 98$ & 24 & 55 & 5 & $7.6 / 8.6$ & 33.7 & $0 / 0$ & $0 / 0$ & $0 / 0$ \\
\hline Moretto et al. [26] & $155 / 77$ & 24 & 54 & 1 & $7.8 / 8.7$ & 31.5 & $0 / 0$ & $0 / 0$ & $0 / 0$ \\
\hline Liutkus et al. [27] & $111 / 54$ & 26 & 54 & 6 & $8.2 / 9.1$ & 33.5 & $0 / 0$ & $0 / 0$ & $0 / 0$ \\
\hline DeFronzo et al. [28] & $223 / 113$ & 30 & 53 & 6 & $8.2 / 9.4$ & 34.0 & $1 / 2$ & $0 / 0$ & $0 / 0$ \\
\hline Buse et al. [29] & $248 / 123$ & 30 & 55 & 6 & $8.6 / 10.3$ & 33.5 & $1 / 2$ & $0 / 0$ & $0 / 0$ \\
\hline Kendall et al. [30] & $486 / 247$ & 30 & 55 & 9 & $8.5 / 9.9$ & 34.0 & $7 / 6$ & $0 / 1$ & $0 / 1$ \\
\hline \multicolumn{10}{|c|}{ Exenatide versus rosiglitazone } \\
\hline DeFronzo $^{\#}$ et al. [24] & $45 / 45$ & 20 & 56 & NR & 7.9/NR & NR & $0 / 0$ & $0 / 0$ & $0 / 0$ \\
\hline \multicolumn{10}{|c|}{ Exenatide versus glibenclamide } \\
\hline Derosa et al. [31] & $63 / 65$ & 52 & 56 & NR & $8.8 / 7.9$ & 28.6 & NR/NR & $0 / 0$ & $0 / 0$ \\
\hline \multicolumn{10}{|c|}{ Exenatide versus BiAsp 30/70 } \\
\hline Bergenstal et al. [32] & $124 / 248$ & 24 & 52 & NR & $10.1 / 11.4$ & 33.8 & NR/NR & $0 / 1$ & $0 / 1$ \\
\hline Nauck et al. [33] & $253 / 248$ & 52 & 58 & 10 & $8.6 / 11.1$ & 30.4 & $10 / 5$ & $2 / 1$ & $1 / 1$ \\
\hline \multicolumn{10}{|c|}{ Exenatide versus glargine } \\
\hline Barnett et al. [34] & $138 / 138$ & 16 & 55 & 7 & $8.9 / 12.0$ & 31.3 & $0 / 0$ & $0 / 0$ & $0 / 0$ \\
\hline NCT00360334 [35] & $118 / 116$ & 26 & 56 & NR & $8.6 / 10.8$ & 34.1 & $2 / 2$ & NR/NR & NR/NR \\
\hline Heine et al. [36] & $282 / 267$ & 26 & 59 & 9 & $8.2 / 10.2$ & 31.3 & $5 / 3$ & $0 / 0$ & $0 / 0$ \\
\hline Bunck et al. [37] & $36 / 33$ & 52 & 58 & 5 & $7.5 / 9.1$ & 30.6 & NR/NR & NR/NR & NR/NR \\
\hline Diamant et al. [38] & $233 / 232$ & 26 & 58 & 8 & $8.3 / 9.8$ & 32.0 & $1 / 0$ & $0 / 0$ & $0 / 0$ \\
\hline \multicolumn{10}{|l|}{ Exenatide versus insulin } \\
\hline Davis et al. [39] & $33 / 16$ & 16 & 53 & 11 & $8.1 / 8.7$ & 34.0 & $1 / 0$ & $0 / 0$ & $0 / 0$ \\
\hline \multicolumn{10}{|c|}{ Exenatide LAR versus placebo } \\
\hline Kim et al. [40] & $30 / 14$ & 15 & 53 & 4 & $8.4 / 10.7$ & 36.0 & $0 / 0$ & $0 / 0$ & $0 / 0$ \\
\hline \multicolumn{10}{|c|}{ Exenatide LAR versus pioglitazone } \\
\hline Bergenstal et al. [41] & $160 / 165$ & 26 & 52 & 6 & $8.5 / 9.1$ & 32.0 & $0 / 3$ & $0 / 0$ & $0 / 0$ \\
\hline \multicolumn{10}{|c|}{ Exenatide LAR versus sitagliptin } \\
\hline Bergenstal et al. [41] ${ }^{\#}$ & $160 / 166$ & 26 & 52 & 6 & $8.5 / 9.1$ & 32.0 & $0 / 1$ & $0 / 1$ & $0 / 0$ \\
\hline \multicolumn{10}{|c|}{ Liraglutide versus placebo } \\
\hline Madsbad et al. [42] & $135 / 29$ & 12 & 57 & 4 & $7.5 / \mathrm{NR}$ & 30.4 & $0 / 0$ & $0 / 0$ & $0 / 0$ \\
\hline Vilsbøll [43] & $123 / 40$ & 14 & 56 & 4 & $8.3 / 11.8$ & 30.1 & $0 / 0$ & $0 / 0$ & $0 / 0$ \\
\hline Seino et al. [44] & $180 / 46$ & 14 & 57 & 8 & $8.3 / \mathrm{NR}$ & 23.9 & $0 / 0$ & $0 / 0$ & $0 / 0$ \\
\hline Kaku et al. [45] & $176 / 88$ & 24 & 60 & 10 & $8.4 / \mathrm{NR}$ & 24.9 & $1 / 1$ & $0 / 0$ & $0 / 0$ \\
\hline Russell-Jones et al. [46] & $232 / 115$ & 26 & 57 & 9 & $8.3 / 9.2$ & 30.6 & $5 / 1$ & $1 / 2$ & $0 / 2$ \\
\hline
\end{tabular}


TABle 3: Continued.

\begin{tabular}{|c|c|c|c|c|c|c|c|c|c|}
\hline Study (ref.) & $\begin{array}{c}\text { Number of } \\
\text { patients } \\
\text { (ID/C) }\end{array}$ & $\begin{array}{c}\text { Trial } \\
\text { duration } \\
\text { (wks) }\end{array}$ & Age (ys) & $\begin{array}{c}\text { Duration of } \\
\text { DM (ys) }\end{array}$ & $\begin{array}{c}\text { HbAlc/FPG } \\
\text { baseline } \\
(\% / \mathrm{mmol} / \mathrm{L})\end{array}$ & $\begin{array}{c}\text { BMI } \\
\text { baseline } \\
\left(\mathrm{Kg} / \mathrm{m}^{2}\right)\end{array}$ & $\begin{array}{l}\text { MACE } \\
(\mathrm{n}, \mathrm{ID} / \mathrm{C})\end{array}$ & $\begin{array}{l}\text { All-cause } \\
\text { mortality } \\
(\mathrm{n}, \mathrm{ID} / \mathrm{C})\end{array}$ & $\begin{array}{c}\text { Cardiovasc. } \\
\text { mortality } \\
(\mathrm{n}, \mathrm{ID} / \mathrm{C}) \\
\end{array}$ \\
\hline Zinman et al. [47] & $355 / 175$ & 26 & 55 & 9 & $8.5 / 10.1$ & 33.7 & $1 / 0$ & $0 / 0$ & $0 / 0$ \\
\hline Marre et al. [48] & $695 / 114$ & 26 & 56 & 6 & $8.4 / 9.7$ & 29.7 & $3 / 2$ & $0 / 0$ & $0 / 0$ \\
\hline Nauck et al. [49] & $724 / 121$ & 26 & 57 & 7 & $8.4 / 10.0$ & 31.2 & $6 / 0$ & $1 / 0$ & $0 / 0$ \\
\hline
\end{tabular}

Liraglutide versus metformin

$\frac{\text { Feinglos et al. [50] 176/34 }}{\text { Liraglutide versus rosiglitazone }}$

Marre $^{\#}$ et al. [48] $\quad 695 / 232 \quad 26 \quad 56$

Liraglutide versus glimepiride

Madsbad" et al. [42]

$135 / 26$

12

57

53

$7.0 / \mathrm{NR}$

34,5

$0 / 0$

$0 / 0$

$0 / 0$

NCT00614120 [35]

$698 / 231$

16

6

$8.4 / 9.7$

29.7

$3 / 0$

$0 / 0$

$0 / 0$

Nauck $^{\#}$ et al. [49]

$724 / 121$

26

57

4

7

7.5/NR

30.4

$0 / 0$

$0 / 0$

$0 / 0$

Garber et al. [51]

498/248

52

53

7

NR/NR

25.5

$3 / 2$

$0 / 0$

$0 / 0$

$7 \quad 8.4 / 10.0$

31.2

$6 / 2$

$1 / 0$

$0 / 0$

Liraglutide versus glibenclamide

NCT00393718 [35] 268/13

Liraglutide versus sitagliptin

Pratley et al. [52]

$446 / 219$

55

5

$8.3 / 9.4$

33.0

$2 / 2$

$0 / 1$

$0 / 0$

Liraglutide versus glargine

Russell-Jones ${ }^{\#}$ et al. [46] 232/23

$26 \quad 57$

57

9

8.3/9.2 $\quad 30.6$

$5 / 1$

$1 / 1$

$0 / 1$

${ }^{\#}$ Studies with multiple comparators; DM: diabetes mellitus; FPG: fasting plasma glucose; MACE: major cardiovascular events; cardiovasc.: cardiovascular; NR: not reported.

In order to reach definitive conclusions on cardiovascular safety of any drug, large-scale, long-term trials should be performed prior to marketing; unfortunately, this effort would be economically unfeasible for pharmaceutical companies. The FDA accepted a compromise, allowing the organization of such trials after drug approval, as a condition for the maintenance of marketing authorization. The limit of this approach is that cardiovascular safety of new drugs will be established only several years after their approval, leaving clinicians without reliable information on this critical point in the meantime.

Meta-analyses of cardiovascular events recorded as adverse events in randomized clinical trials designed for other purposes can represent an additional source of information. This approach has several limitations, most notably the lack of predefined diagnostic criteria and screening methods for incident cardiovascular disease, with the risk of misdiagnosis and underdiagnosis. It should also be recognized that in some of the trials included, cardiovascular events were reported only as adverse events, without being prospectively adjudicated. Moreover, the limited duration of trials designed for metabolic purposes can impair their ability to detect longer-term effects on atherogenesis. Furthermore, the meta-analysis of small trials with few events each poses some specific, and complex, statistical problems [17]. All these limitations affected the reliability of results of some meta-analyses $[6,7]$ on cardiovascular safety of hypoglycemic drugs $[10,17,18]$.

Those considerations should be taken into account when interpreting the results of the present meta-analysis, which exclude, at least in the short term, any major adverse effect of GLP-1 receptor agonists on cardiovascular morbidity. Interestingly, those drugs, as a class, are below to the 1.3 threshold chosen by the FDA for the upper limit of $95 \%$ confidence interval to establish the cardiovascular safety of a new drug.

Interestingly, a significant reduction of cardiovascular morbidity with GLP-1 receptor agonists was observed in comparison with placebo. This result should be discussed with great caution, considering the limitations highlighted above; in fact, a meta-analysis of trials performed for different (noncardiovascular) endpoints provides reliable information on safety, but not on efficacy. Speculatively, several mechanisms could underlie a beneficial effect of GLP-1 receptor agonists on cardiovascular risk. Reduction of blood glucose, body weight, and blood pressure, as well as favorable effects on lipid profile, have all been reported. Direct myocardial effects of GLP-1 receptor stimulation could theoretically reduce the functional impact of myocardial ischemia [13], leading to clinical improvements. However, the possibility of a beneficial action of GLP-1 receptor agonists on cardiovascular events should be confirmed through specifically designed randomized clinical trials. 
In conclusion, GLP-1 receptor agonists do not appear to increase cardiovascular morbidity in comparison with placebo or other active drugs. Any possible beneficial action should be assessed in further trials.

\section{Author Contributions}

M. Monami organized the collection of clinical data, prepared and revised the paper, and performed data analysis. F. Cremasco collected clinical data and assisted in study design and data analysis. C. Lamanna collected clinical data and revised the paper. C. Colombi collected clinical data and assisted in study design. S. Zannoni collected clinical data. I. Iacomelli collected clinical data N. Marchionni reviewed/edited the paper. E. Mannucci designed the study, prepared and revised the paper, and took part in data analysis.

\section{Conflict of Interests}

The corresponding author confirms that he had full access to all the data in the study and had final responsibility for the decision to submit for publication. M. Monami has received speaking fees from Eli Lilly and Sanofi-Aventis. F. Cremasco is currently employed by Eli Lilly. N. Marchionni has received speaking fees from Eli Lilly, Novo Nordisk, and SanofiAventis, and research grants from Eli Lilly, Novo Nordisk, and Sanofi-Aventis. E. Mannucci has received consultancy fees from Eli Lilly and Novo Nordisk, speaking fees from Eli Lilly, Novo Nordisk, and Sanofi-Aventis, and research grants from Eli Lilly, Novo Nordisk, and Sanofi-Aventis.

\section{Acknowledgments}

The authors would like to thank the following persons who were involved in the organization of the study and in the collection and management of data: Ilaria Bracali, Rossella Del Bianco, Cristina Marchi, and Maria Vivarelli.

\section{References}

[1] The University Group Diabetes Program, "A study of the effects of hypoglycemic agents on vascular complications in patients with adult-onset diabetes. V. Evaluation of pheniformin therapy," Diabetes, vol. 24, supplement 1, pp. 65-184, 1975.

[2] A. D. Rao, N. Kuhadiya, K. Reynolds, and V. A. Fonseca, "Is the combination of sulfonylureas and metformin associated with an increased risk of cardiovascular disease or all- cause mortality? A meta-analysis of observational studies," Diabetes Care, vol. 31, no. 8, pp. 1672-1678, 2008.

[3] S. Finfer, R. Bellomi, D. Blair et al., "Intensive versus conventional glucose control in critically Ill patients," The New England Journal of Medicine, vol. 360, no. 13, pp. 1283-1297, 2009.

[4] K. Malmberg, L. Rydén, H. Wedel et al., "FASTTRACK intense metabolic control by means of insulin in patients with diabetes mellitus and acute myocardial infarction (DIGAMI 2): effects on mortality and morbidity," European Heart Journal, vol. 26, no. 7, pp. 650-661, 2005.
[5] R. M. Lago, P. P. Singh, and R. W. Nesto, "Congestive heart failure and cardiovascular death in patients with prediabetes and type 2 diabetes given thiazolidinediones: a meta-analysis of randomised clinical trials," Lancet, vol. 370, no. 9593, pp. 1129-1136, 2007.

[6] S. E. Nissen and K. Wolski, "Effect of rosiglitazone on the risk of myocardial infarction and death from cardiovascular causes," The New England Journal of Medicine, vol. 356, no. 24, pp. 2457-2471, 2007.

[7] S. Singh, Y. K. Loke, and C. D. Furberg, "Long-term risk of cardiovascular events with rosiglitazone: a meta-analysis," Journal of the American Medical Association, vol. 298, no. 10, pp. 1189-1195, 2007.

[8] "Effect of intensive blood-glucose control with metformin on complications in overweight patients with type 2 diabetes (UKPDS 34). UK Prospective Diabetes Study (UKPDS) Group," The Lancet, vol. 352, pp. 854-865, 1998.

[9] P. D. Home, S. J. Pocock, H. Beck-Nielsen et al., "Rosiglitazone evaluated for cardiovascular outcomes - an interim analysis," The New England Journal of Medicine, vol. 357, no. 1, pp. 2838, 2007.

[10] E. Mannucci, M. Monami, and N. Marchionni, "Rosiglitazone and cardiovascular risk," The New England Journal of Medicine, vol. 357, no. 9, pp. 938-940, 2007.

[11] E. Mannucci, M. Monami, M. Di Bari et al., "Cardiac safety profile of rosiglitazone: a comprehensive meta-analysis of randomized clinical trials," International Journal of Cardiology, vol. 143, no. 2, pp. 135-140, 2010.

[12] E. Mannucci and C. M. Rotella, "Future perspectives on glucagon-like peptide-1, diabetes and cardiovascular risk," Nutrition, Metabolism and Cardiovascular Diseases, vol. 18, no. 9, pp. 639-645, 2008.

[13] G. G. Sokos, L. A. Nikolaidis, S. Mankad, D. Elahi, and R. P. Shannon, "Glucagon-like peptide-1 infusion improves left ventricular ejection fraction and functional status in patients with chronic heart failure," Journal of Cardiac Failure, vol. 12, no. 9, pp. 694-699, 2006.

[14] A. R. Jadad, R. A. Moore, D. Carroll et al., "Assessing the quality of reports of randomized clinical trials: is blinding necessary?" Controlled Clinical Trials, vol. 17, no. 1, pp. 1-12, 1996.

[15] D. B. Petitti, "Approaches to heterogeneity in meta-analysis," Statistics in Medicine, vol. 20, no. 23, pp. 3625-3633, 2001.

[16] D. Moher, A. Liberati, J. Tetzlaff et al., "Preferred reporting items for systematic reviews and meta-analyses: the PRISMA statement," Annals of Internal Medicine, vol. 151, no. 4, pp. 264-269, 2009.

[17] G. A. Diamond, L. Bax, and S. Kaul, "Uncertain effects of rosiglitazone on the risk for myocardial infarction and cardiovascular death," Annals of Internal Medicine, vol. 147, no. 8, pp. 578-581, 2007.

[18] J. J. Shuster, L. S. Jones, and D. A. Salmon, "Fixed vs random effects meta-analysis in rare event studies: the Rosiglitazone link with myocardial infarction and cardiac death," Statistics in Medicine, vol. 26, no. 24, pp. 4375-4385, 2007.

[19] J. Rosenstock, J. Reusch, M. Bush, F. Yang, and M. Stewart, "Potential of albiglutide, a long-acting GLP-1 receptor agonist, in type 2 diabetes: a randomized controlled trial exploring weekly, biweekly, and monthly dosing," Diabetes Care, vol. 32, no. 10, pp. 1880-1886, 2009.

[20] A. Gill, B. J. Hoogwerf, J. Burger et al., "Effect of exenatide on heart rate and blood pressure in subjects with type 2 diabetes mellitus: a double-blind, placebo-controlled, randomized pilot study," Cardiovascular Diabetology, vol. 9, article 6, 2010. 
[21] T. Kadowaki, M. Namba, A. Yamamura, H. Sowa, A. M. Wolka, and R. G. Brodows, "Exenatide exhibits dose-dependent effects on glycemic control over 12 weeks in Japanese patients with suboptimally controlled type 2 diabetes," Endocrine Journal, vol. 56, no. 3, pp. 415-424, 2009.

[22] B. Zinman, B. J. Hoogwerf, S. Durán García et al., "The effect of adding exenatide to a thiazolidinedione in suboptimally controlled type 2 diabetes: a randomized trial," Annals of Internal Medicine, vol. 146, no. 7, pp. 477-485, 2007.

[23] Y. Gao, K. H. Yoon, L. M. Chuang et al., "Efficacy and safety of exenatide in patients of Asian descent with type 2 diabetes inadequately controlled with metformin or metformin and a sulphonylurea," Diabetes Research and Clinical Practice, vol. 83, no. 1, pp. 69-76, 2009.

[24] R. A. DeFronzo, C. Triplitt, Y. Qu, M. S. Lewis, D. Maggs, and L. C. Glass, "Effects of exenatide plus rosiglitazone on $\beta$-cell function and insulin sensitivity in subjects with type 2 diabetes on metformin," Diabetes Care, vol. 33, no. 5, pp. 951-957, 2010.

[25] C. M. Apovian, R. M. Bergenstal, R. M. Cuddihy et al., "Effects of exenatide combined with lifestyle modification in patients with type 2 diabetes," American Journal of Medicine, vol. 123, no. 5, pp. 468.e9-468.e17, 2010.

[26] T. J. Moretto, D. R. Milton, T. D. Ridge et al., "Efficacy and tolerability of exenatide monotherapy over 24 weeks in antidiabetic drug-naive patients with type 2 diabetes: a randomized, double-blind, placebo-controlled, parallel-group study," Clinical Therapeutics, vol. 30, no. 8, pp. 1448-1460, 2008.

[27] J. Liutkus, J. Rosas Guzman, P. Norwood et al., "A placebocontrolled trial of exenatide twice-daily added to thiazolidinediones alone or in combination with metformin," Diabetes, Obesity and Metabolism, vol. 12, no. 12, pp. 1058-1065, 2010.

[28] R. A. DeFronzo, R. E. Ratner, J. Han, D. D. Kim, M. S. Fineman, and A. D. Baron, "Effects of exenatide (exendin-4) on glycemic control and weight over 30 weeks in metformintreated patients with type 2," Diabetes Care, vol. 28, no. 5, pp. 1092-1100, 2005.

[29] J. B. Buse, R. R. Henry, J. Han, D. D. Kim, M. S. Fineman, and A. D. Baron, "Effects of exenatide (exendin-4) on glycemic control over 30 weeks in sulfonylurea-treated patients with type 2 diabetes," Diabetes Care, vol. 27, no. 11, pp. 2628-2635, 2004.

[30] D. M. Kendall, M. C. Riddle, J. Rosenstock et al., "Effects of exenatide (exendin-4) on glycemic control over 30 weeks in patients with type 2 diabetes treated with metformin and a sulfonylurea," Diabetes Care, vol. 28, no. 5, pp. 1083-1091, 2005.

[31] G. Derosa, P. Maffioli, S. A. T. Salvadeo et al., "Exenatide versus glibenclamide in patients with diabetes," Diabetes Technology and Therapeutics, vol. 12, no. 3, pp. 233-240, 2010.

[32] R. Bergenstal, A. Lewin, T. Bailey, D. Chang, T. Gylvin, and V. Roberts, "Efficacy and safety of biphasic insulin aspart 70/30 versus exenatide in subjects with type 2 diabetes failing to achieve glycemic control with metformin and a sulfonylurea," Current Medical Research and Opinion, vol. 25, no. 1, pp. 6575, 2009.

[33] M. A. Nauck, S. Duran, D. Kim et al., "A comparison of twice-daily exenatide and biphasic insulin aspart in patients with type 2 diabetes who were suboptimally controlled with sulfonylurea and metformin: a non-inferiority study," Diabetologia, vol. 50, no. 2, pp. 259-267, 2007.
[34] A. H. Barnett, J. Burger, D. Johns et al., "Tolerability and efficacy of exenatide and titrated insulin glargine in adult patients with type 2 diabetes previously uncontrolled with metformin or a sulfonylurea: a multinational, randomized, open-label, two-period, crossover noninferiority trial," Clinical Therapeutics, vol. 29, no. 11, pp. 2333-2348, 2007.

[35] http://www.clinicaltrials.gov/, 2010.

[36] R. J. Heine, L. F. Van Gaal, D. Johns, M. J. Mihm, M. H. Widel, and R. G. Brodows, "Exenatide versus insulin glargine in patients with suboptimally controlled type 2 diabetes: a randomized trial," Annals of Internal Medicine, vol. 143, no. 8, pp. 559-569, 2005.

[37] M. C. Bunck, M. Diamant, A. Cornér et al., "One-year treatment with exenatide improves $\beta$-cell function, compared with insulin glargine, in metformin-treated type 2 diabetic patients: a randomized, controlled trial," Diabetes Care, vol. 32, no. 5, pp. 762-768, 2009.

[38] M. Diamant, L. Van Gaal, S. Stranks et al., "Once weekly exenatide compared with insulin glargine titrated to target in patients with type 2 diabetes (DURATION-3): an open-label randomised trial," The Lancet, vol. 375, no. 9733, pp. 22342243, 2010.

[39] S. N. Davis, D. Johns, D. Maggs, H. Xu, J. H. Northrup, and R. G. Brodows, "Exploring the substitution of exenatide for insulin in patients with type 2 diabetes treated with insulin in combination with oral antidiabetes agents," Diabetes Care, vol. 30, no. 11, pp. 2767-2772, 2007.

[40] D. Kim, L. MacConell, D. Zhuang et al., "Effects of onceweekly dosing of a long-acting release formulation of exenatide on glucose control and body weight in subjects with type 2 diabetes," Diabetes Care, vol. 30, no. 6, pp. 1487-1493, 2007.

[41] R. M. Bergenstal, C. Wysham, L. MacConell et al., "Efficacy and safety of exenatide once weekly versus sitagliptin or pioglitazone as an adjunct to metformin for treatment of type 2 diabetes (DURATION-2): a randomised trial," The Lancet, vol. 376, pp. 431-439, 2010.

[42] S. Madsbad, O. Schmitz, J. Ranstam, G. Jakobsen, and D. R. Matthews, "Improved glycemic control with no weight increase in patients with type 2 diabetes after once-daily treatment with the long-acting glucagon-like peptide 1 analog liraglutide (NN2211): a 12-week, double-blind, randomized, controlled trial," Diabetes Care, vol. 27, no. 6, pp. 1335-1342, 2004.

[43] T. Vilsbøll, "Liraglutide: a once-daily GLP-1 analogue for the treatment of Type 2 diabetes mellitus," Expert Opinion on Investigational Drugs, vol. 16, no. 2, pp. 231-237, 2007.

[44] Y. Seino, M. F. Rasmussen, M. Zdravkovic, and K. Kaku, "Dose-dependent improvement in glycemia with once-daily liraglutide without hypoglycemia or weight gain: a doubleblind, randomized, controlled trial in Japanese patients with type 2 diabetes," Diabetes Research and Clinical Practice, vol. 81, no. 2, pp. 161-168, 2008.

[45] K. Kaku, M. F. Rasmussen, P. Clauson, and Y. Seino, "Improved glycaemic control with minimal hypoglycaemia and no weight change with the once-daily human glucagonlike peptide-1 analogue liraglutide as add-on to sulphonylurea in Japanese patients with type 2 diabetes," Diabetes, Obesity and Metabolism, vol. 12, no. 4, pp. 341-347, 2010.

[46] D. Russell-Jones, A. Vaag, O. Schmitz et al., "Liraglutide vs insulin glargine and placebo in combination with metformin and sulfonylurea therapy in type 2 diabetes mellitus (LEAD5 met+SU): a randomised controlled trial," Diabetologia, vol. 52, no. 10, pp. 2046-2055, 2009. 
[47] B. Zinman, J. Gerich, J. B. Buse et al., "Efficacy and safety of the human glucagon-like peptide-1 analog liraglutide in combination with metformin and thiazolidinedione in patients with type 2 diabetes (LEAD-4 Met + TZD)," Diabetes Care, vol. 32, pp. 1224-1230, 2010.

[48] M. Marre, J. Shaw, M. Brändle et al., "Liraglutide, a oncedaily human GLP-1 analogue, added to a sulphonylurea over 26 weeks produces greater improvements in glycaemic and weight control compared with adding rosiglitazone or placebo in subjects with Type 2 diabetes (LEAD-1 SU)," Diabetic Medicine, vol. 26, no. 3, pp. 268-278, 2009.

[49] M. Nauck, A. Frid, K. Hermansen et al., "Efficacy and safety comparison of liraglutide, glimepiride, and placebo, all in combination with metformin, in type 2 diabetes: the LEAD (liraglutide effect and action in diabetes)-2 study," Diabetes Care, vol. 32, pp. 84-90, 2009.

[50] M. N. Feinglos, M. F. Saad, F. X. Pi-Sunyer, B. An, and O. Santiago, "Effects of liraglutide (NN2211), a long-acting GLP1 analogue, on glycaemic control and bodyweight in subjects with Type 2 diabetes," Diabetic Medicine, vol. 22, no. 8, pp. 1016-1023, 2005.

[51] A. Garber, R. Henry, R. Ratner et al., "Liraglutide versus glimepiride monotherapy for type 2 diabetes (LEAD3 Mono): a randomised, 52-week, phase III, double-blind, parallel-treatment trial," The Lancet, vol. 373, no. 9662, pp. 473-481, 2009.

[52] R. E. Pratley, M. Nauck, T. Bailey et al., "Liraglutide versus sitagliptin for patients with type 2 diabetes who did not have adequate glycaemic control with metformin: a 26-week, randomised, parallel-group, open-label trial," Lancet, vol. 375, no. 9724 , pp. $1447-1456,2010$. 


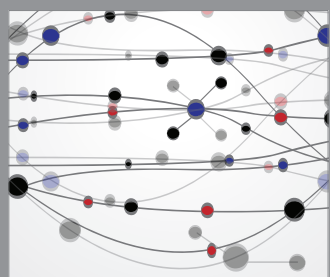

The Scientific World Journal
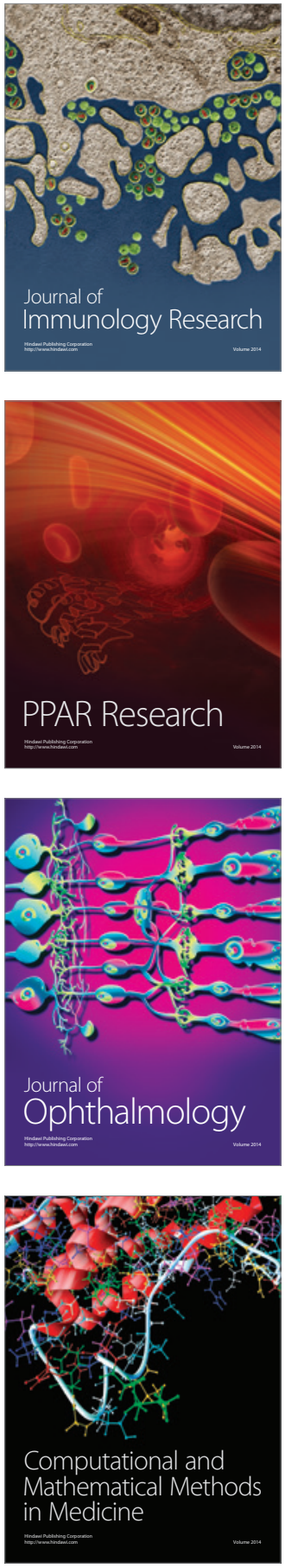

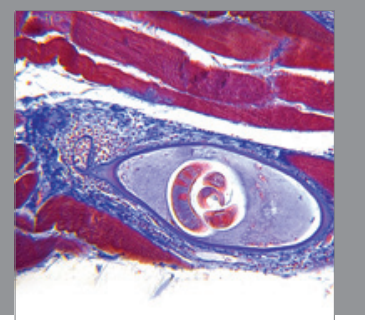

Gastroenterology

Research and Practice
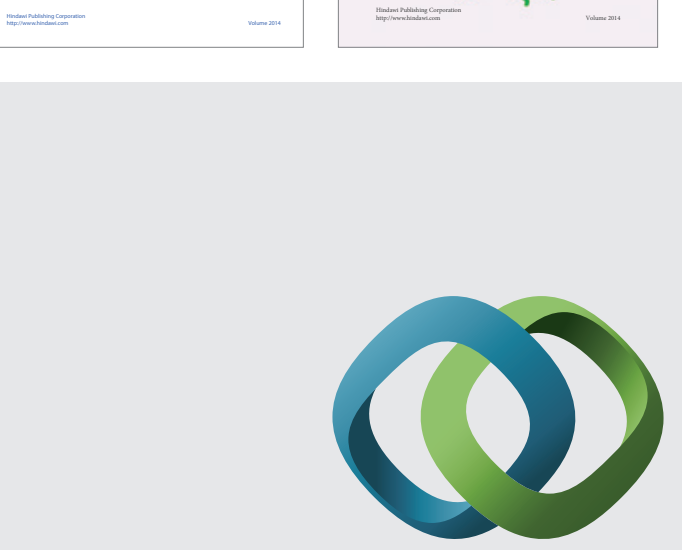

\section{Hindawi}

Submit your manuscripts at

http://www.hindawi.com
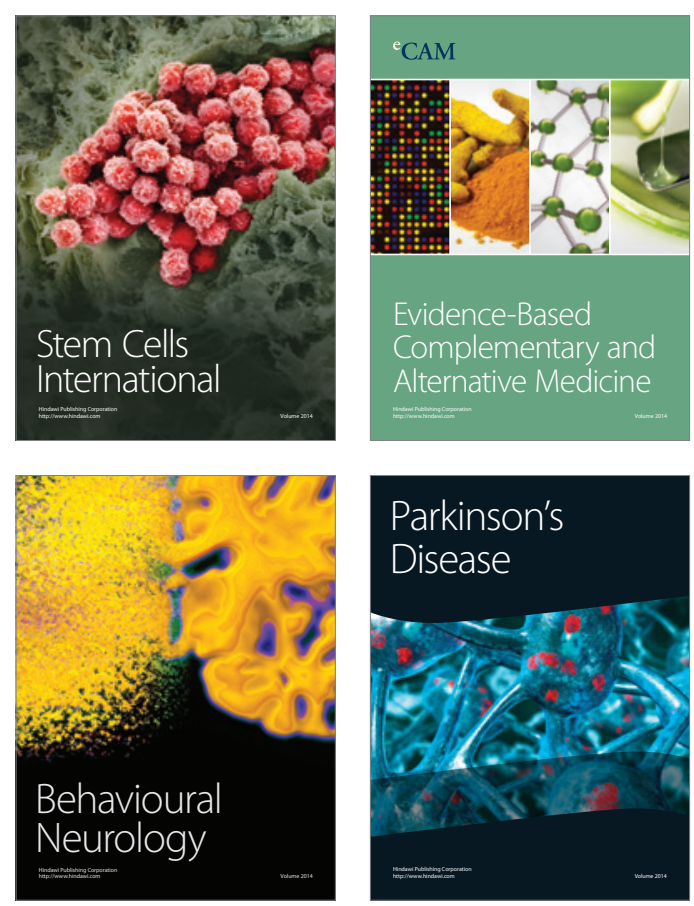

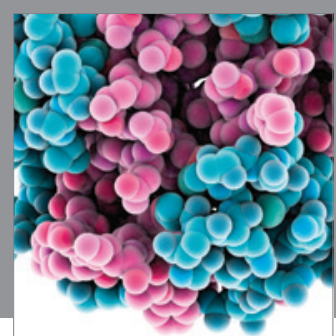

Journal of
Diabetes Research

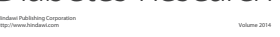

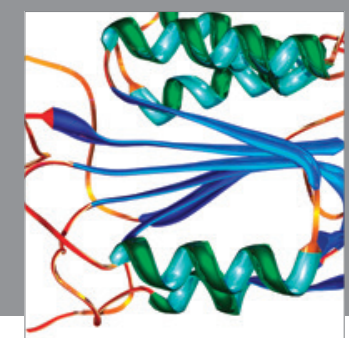

Disease Markers
